\title{
Bilateral leukocoria in infant with afibrinogenemia
}

\author{
M Necati Demir' \\ Mehmet Akif Acar' \\ Yusuf Ziya Aral ${ }^{2}$ \\ Nurten Ünlü'
}

'Ankara Education and Research Hospital, Department of Ophthalmology, Ankara, Turkey; ${ }^{2}$ Aydin Menderes University, Faculty of Medicine, Department of Pediatric Hematology, Aydin, Turkey
Correspondence: Nurten Ünlü Tunalı Hilmi Cad. 33/8, Küçükesat 06660 Ankara, Turkey

$\mathrm{Tel}+903125953482$

$\mathrm{Fax}+903124339600$

Email unlunurten@yahoo.com
Purpose: To report a bilateral leukocoria case in a patient suffering from afibrinogenemia. Methods: An observational case where congenital afibrinogenemia was presented with bilateral retinal and vitreous hemorrhages that proceeded to vitreoretinal surgery was presented. In addition, complete ophthalmic and radiological examinations and vitreoretinal surgery were performed.

Results: Right eye had a complete recovery while the left eye showed serious proliferative vitreoretinopathy and shortened retina. Three years after the surgery clinical examination showed that the right eye was aphacic with an attached retina and clear ocular media while the left eye was phtysic.

Conclusion: We recommend broad clotting profile for infants suffering from vitreous or retinal hemorrhages with no obvious physical abuse. Our present case furthermore implies that afibrinogenemia can lie beneath the pathogenesis of bilateral leukocoria and should alert physician for the presence of an afibrinogenemia among several types of bleeding predispositions.

Keywords: afibrinogenemia, retinal hemorrhage, vitreous hemorrhage, vitrectomy

\section{Introduction}

Retinal hemorrhage in infants is shown to be associated with systemic and several other ocular pathologic factors including intracranial disorders, hematologic disturbances, chorioretinal infections, and retinal vascular diseases (Emerson et al 2001). Intraretinal hemorrhages can also occur in association with shaken baby syndrome. Congenital afibrinogenemia is a rare autosomal-recessive hemorrhagic disorder characterized by markedly reduced or undetectable levels of fibrinogen in the blood (Marshman et al 1999).

There are few studies indicating blood dyscrasias as a cause of retinal and vitreous hemorrhages in neonates (Marshman et al 1999). In the current literature, to the best of our knowledge, this is the first case regarding an afibrinogenemia patient who had bilateral retinal and vitreous hemorrhages that proceeded to vitreoretinal surgery.

\section{Case report}

A three-month-old boy was admitted to our ophthalmology department for the evaluation of bilateral leukocoria that was noted one month earlier. He was born at term by normal vaginal delivery and his weight was $3040 \mathrm{~g}$. Following an afebril convulsion attack 10 days after the birth, he was diagnosed with congenital afibrinogenemia. The prothrombin and partial thromboplastin times were prolonged and plasma fibrinogen levels were undetectable (less than $10 \mathrm{mg} / \mathrm{dl}$ ). Furthermore, his hemoglobin concentration was $11.4 \mathrm{~g} / \mathrm{dl}$. Computed tomography (CT) scans and MRI showed intravitreal hemorrhage and subdural hematomas in the brain (Figure 1). The patient was negative for infectious agents and his thyroid function and thyroid-stimulating hormone levels in serum were found to be normal. In addition to the presence of shallow anterior 
A

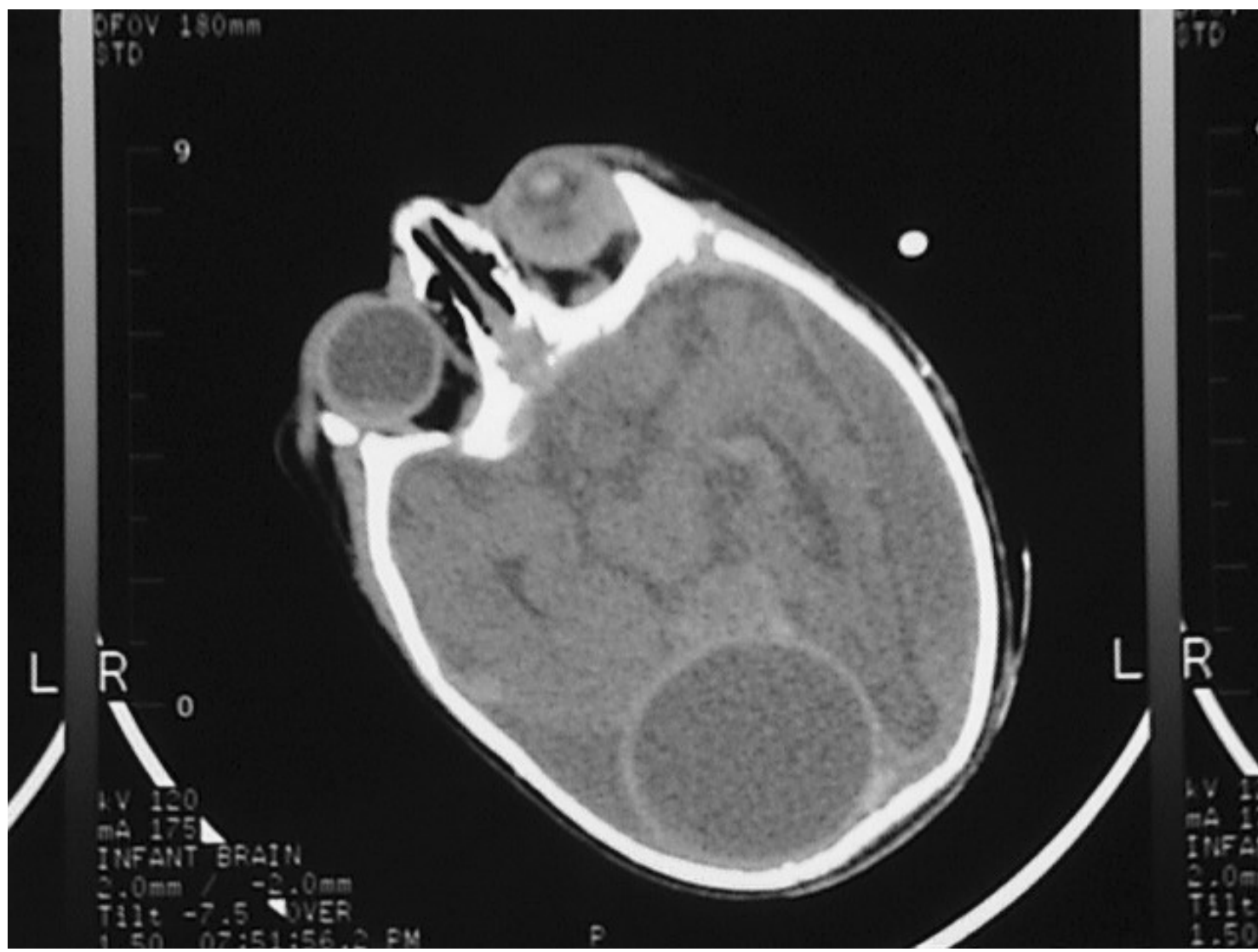

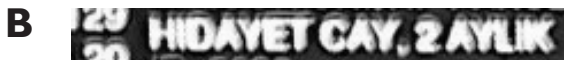

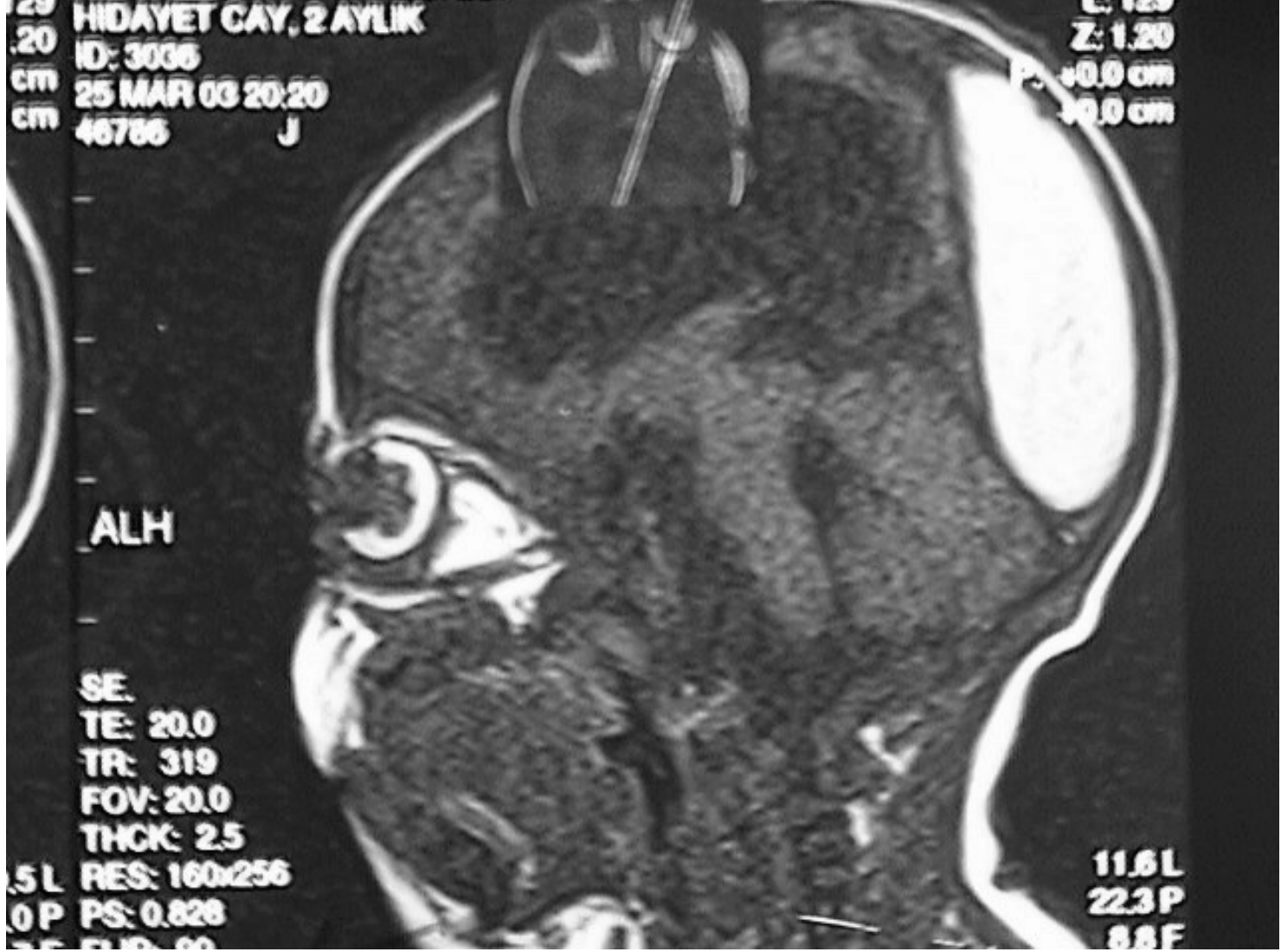

Figure I.A Computed tomography scans of the brain. B. Magnetic resonance image showing subdural hematomas and intravitreal hemorrhage. 

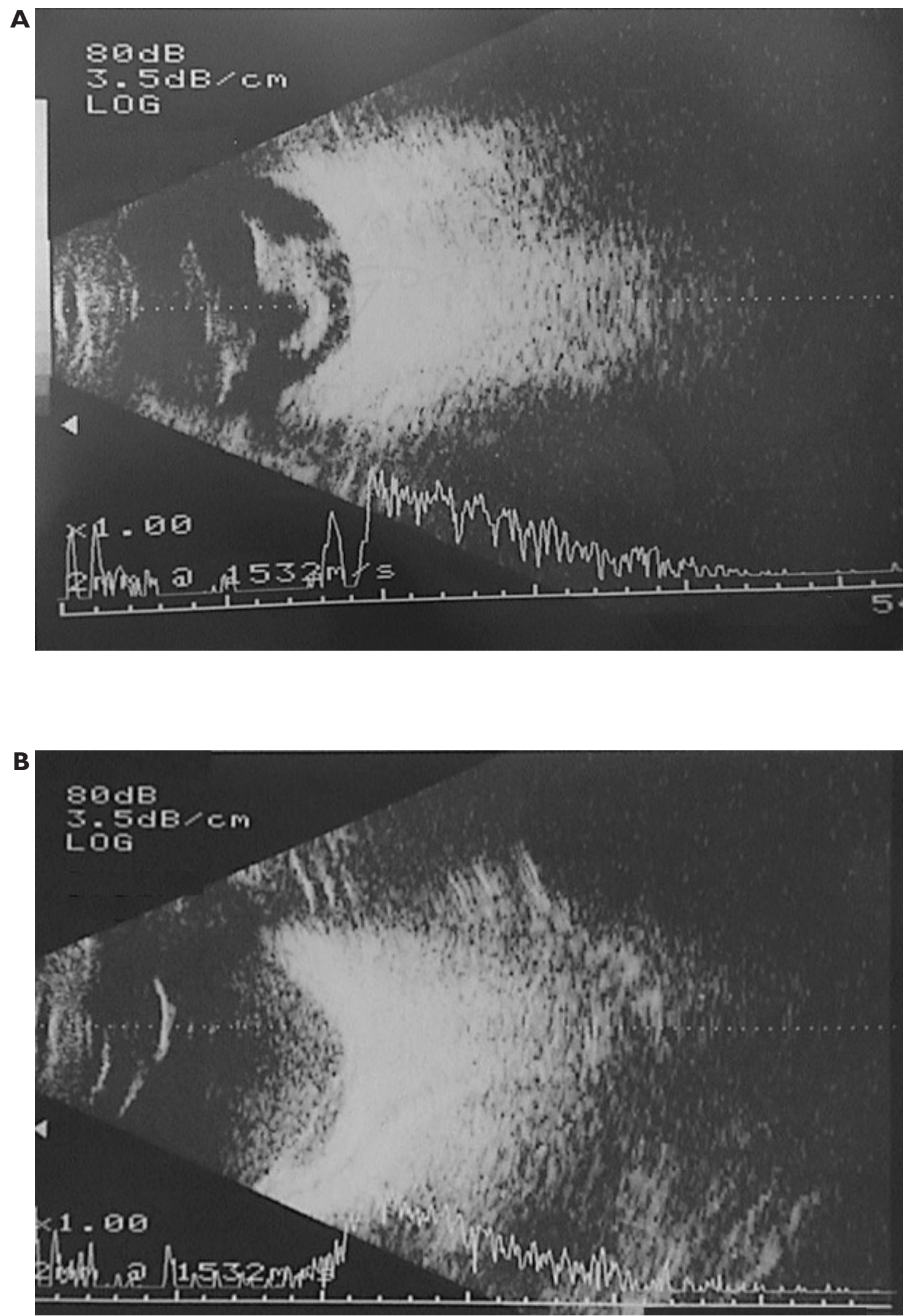

Figure 2.A B scan ultrasonography of the right eye:Vitreous hemorrhage. B. B scan ultrasonography of the left eye:Vitreous hemorrhage and retinal traction. 
chambers and cataractous lenses, dilated fundus examination revealed dense bilateral vitreous hemorrhages. Moreover, B-scan ultrasonography showed bilateral dense vitreous opacities and retinal tractions in the left eye (Figure 2). Both eyes were positive for light reaction. Family histories disclosed that the parents were second cousins and the baby was their first child. Family history did not disclose any bleeding disorders.

Prior to vitrectomy, pre-operative fibrinogen levels were maintained at $150 \mathrm{mg} / \mathrm{dl}$ through fresh frozen plasma infusion. Lensectomy and vitrectomy of the left eye were performed first. The entry was completed via the pars plicata, immediately posterior to the iris root. Serious proliferative vitreoretinopathy with many starfolds, preretinal and subretinal membranes, and cyclytic membrane were present and it was impossible to reattach the shortened retina. Two weeks after the first operation, lensectomy and vitrectomy of the right eye were also completed.

Postoperatively contact lens was given to the right eye to treat afakia. As the left eye was phtysic no further ortoptic treatment was given besides contact lens.

Three years after the operation, the examination of the patient revealed that the left eye had phtysis while the right eye was aphacic with an attached retina and clear ocular media in addition to a visual acuity of counting figures at 2 meters.

\section{Discussion}

The incidence of retinal hemorrhage in newborns shows a wide range between $2.6 \%$ to $50.0 \%$ (Marshman et al 1999; Emerson et al 2001). The incidence of hemorrhages is higher for the infants born with the help of induced labor. Approximately $90 \%$ of intraretinal hemorrhages detected at birth resolves within 2 weeks and becomes undetectable by 4 weeks after birth (Buys et al 1992; Emerson et al 2001). Retinal hemorrhages in newborns of induced labor also resolve by 5 weeks of age. Emerson and colleagues (2001) reported that intraretinal hemorrhage detected in infants older than 1 month is not likely to be related to birth, a finding that may be useful in the evaluation of suspected physical abuse or other ocular and systemic diseases.

Retinal hemorrhages are shown to occur frequently in blood dyscrasias such as pancytopenia, megaloblastic anemia, coagulopathies, and hyperviscosity and these are reported to be more common in adults than children (Kaur and Taylor 1992; Sheridan and Byron 1992). Other specific clotting deficiency such as protein $\mathrm{C}$ deficiency in homozygous individuals is reported to be the cause of intravitreal hemorrhage in infants (Pulido et al 1987). Furthermore, several other types of bleeding diathesis should be considered among the spontaneous grounds for retinal hemorrhage. For instance, fibrinogen level is a critical factor for bleeding diathesis. Marshman and colleagues (1999) reported a premature baby who suffered from a low plasma fibrinogen level had bilateral retinal hemorrhages that proceeded to vitreous hemorrhage.

Studies show that while $5 \%$ to $23 \%$ of physically abused children endure from retinal hemorrhage, the incidence of retinal hemorrhages is much higher (50\% to $100 \%)$ in patients with shaken baby syndrome (Buys et al 1992). Therefore, a discrepancy in diagnosis of retinal and vitreous hemorrhages in infants should always prompt physician for a child abuse or shaken baby syndrome. These hemorrhages often involve the posterior pole in the nerve fibers and intraretinal layers and they are bilateral in 58\% to $100 \%$ of cases. The receding of retinal hemorrhages varies greatly, resolving anywhere from 10 days to several months (Buys et al 1992).

To the best of our knowledge, the present case is the first case reported in the literature regarding a normal term baby with afibrinogenemia who underwent a vitrectomy and lensectomy for bilateral vitreous hemorrhage as indicated CT scan showing a unilateral retinal detachment and intracranial hemorrhage.

Congenital afibrinogenemia is a rare autosomal disorder in which the patients endure hemorrhages due to impaired clot formation and markedly reduced platelet function (Fernando and Dharmasena 1957). Severe hemorrhages are reported following trauma although spontaneous bleeding tendency is usually mild to moderate in these patients. In the present case, our clinical examination and anamnesis revealed no sign of child abuse. Thus the undetectable fibrinogen level seems to be the major cause for the intracranial, retinal and vitreous hemorrhages for the child.

Treatment of afibrinogenemia consists of administration of cryoprecipitate or other plasma products containing fibrinogen to maintain post infusion fibrinogen level, at least, at 75 to $100 \mathrm{mg} / \mathrm{dl}$. In the present case, cryoprecipitate was given before surgery and no hemorrhage disorder was detected during the surgery. In the right eye, there was severe vitreous hemorrhage with retinal detachment and it was not possible to reattach the retina; whereas, the surgery was successful in the left eye with clear ocular media and attached retina. This baby's presentation could be easily mistaken for that of shaken baby syndrome if the hematological data were not documented and evaluated. We recommend extensive 
clotting profiling of children who present with vitreous or retinal hemorrhage.

In conclusion, our present case suggests that afibrinogenemia is a potential factor underlying in the pathogenesis of bilateral leukocoria and should prompt physician for the presence of an afibrinogenemia among several types of bleeding diathesis at the absence of physical abuse.

\section{References}

Buys YM, Levin AV, Enzenauer RW, et al. 1992. Retinal findings after head trauma in infants and young children. Ophthalmology, 99:1718-23.
Emerson MV, Pieramici DJ, Stoessel KM, et al. 2001. Incidence and rate of disappearance of retinal hemorrhage in newborns. Ophthalmology, 108:36-9.

Fernando PB, Dharmasena BD. 1957. A case of congenital afibrinogenemia. Blood, 5:474-9.

Kaur B, Taylor D. 1992. Fundus hemorrhages in infancy. Surv Ophthalmol, 37:1-17.

Marshman WE, Adams GG, Ohri R. 1999. bilateral vitreous hemorrhages in an infant with low fibrinogen levels. J AAPOS, 3:255-6.

Pulido JS, Lingua RW, Cristol S, et al. 1987. Protein C deficiency associated with vitreous hemorrhage in a neonate. Am J Ophthalmol, 104:546-7.

Sheridan L, Byron L. 1992. Bilateral retinal hemorrhages from megaloblastic anemia: case report and review of literature. Ann Ophthalmol, 24:86-90. 
